\title{
Cryogenic magnetic bearing scanning mechanism design for the SPICA/SAFARI Fourier Transform Spectrometer
}

\author{
Teun C. van den Dool ${ }^{* a}$, Roger F.M.M. Hamelinck ${ }^{\mathrm{a}}$, Bob Kruizinga ${ }^{\mathrm{a}}$, Wim L.M. Gielesen ${ }^{\mathrm{a}}$, \\ Ben C. Braam ${ }^{a}$, Jan R. Nijenhuis ${ }^{a}$, Nicolas Loix ${ }^{b}$, Stanislas Luyckx ${ }^{b}$, Dennis van Loon ${ }^{c}$, \\ Peter Paul Kooijman ${ }^{c}$, Bruce M. Swinyard ${ }^{\mathrm{d}}$ \\ ${ }^{a}$ TNO S\&I, Stieltjesweg 1, 2628 CK Delft, Netherlands. \\ ${ }^{b}$ Micromega Dynamics SA, Rue du Trou du Sart 10, B-5380 Fernelmont, Belgium. \\ ${ }^{\mathrm{c}} \mathrm{SRON}$, Sorbonnelaan 2, 3584 CA Utrecht, Netherlands. \\ ${ }^{\mathrm{d}}$ RAL-Space Science and Technology Department, R25 1.92, Chilton, Didcot, OX11 0QX, Oxfordshire, UK.
}

\begin{abstract}
TNO, together with its partners Micromega and SRON, have designed a cryogenic scanning mechanism for use in the SAFARI Fourier Transform Spectrometer (FTS) on board of the SPICA mission.

The optics of the FTS scanning mechanism (FTSM) consists of two back-to-back cat's-eyes. The optics are mounted on a central "back-bone" tube which houses all the important mechatronic parts: the magnetic bearing linear guiding system, a magnetic linear motor serving as the OPD actuator, internal metrology with nanometer resolution, and a launch lock.

A magnetic bearing is employed to enable a large scanning stroke in a small volume. It supports the optics in a freefloating way with no friction, or other non-linearities, enabling sub-nanometer accuracy within a single stage with a stroke of $-4 \mathrm{~mm}$ to $+31.5 \mathrm{~mm}$.

Because the FTSM will be used at cryogenic temperatures of 4 Kelvin, the main structure and optics are all constructed from 6061 Aluminum. The overall outside dimensions of the FTSM are: $393 \times 130 \times 125 \mathrm{~mm}$, and the mass is $2.2 \mathrm{~kg}$.
\end{abstract}

Keywords: SPICA, SAFARI, Fourier Transform Spectrometer, optical delay line, active magnetic bearings, cryogenic, fiber interferometer.

\section{INTRODUCTION}

SPICA (SPace Infrared telescope for Cosmology and Astrophysics, see Figure 1) is the proposed next generation space infrared observatory. The mission will study formation of planets, solar system processes, and the origin of the universe. SPICA is an international project, led by the Japanese space agency JAXA, with contributions from Europe. SPICA has been selected as a candidate ESA M-class Cosmic Vision mission.

SPICA will have a single $3.5 \mathrm{~m}$ mirror operating at 4.5 Kelvin. The wavelength range will cover 5 to $210 \mu \mathrm{m}$. Five focal plane instruments are anticipated:

- $\quad$ a large-format MIR camera $(5-38 \mu \mathrm{m})$

- $\quad$ a MIR spectrometer (R 30,000 at 4-18 $\mu \mathrm{m} ; \mathrm{R} \sim 3,000$ at 16-38 $\mu \mathrm{m})$

- $\quad$ a high contrast (1e-6) MIR coronagraph (5-27 $\mu \mathrm{m})$ with photometric and spectroscopic capability

- $\quad$ a single-pixel high sensitivity, low-resolution FIR/sub-mm grating spectrometer $(40-350 \mu \mathrm{m})$

- $\quad$ a FIR imaging spectrometer and camera $(35-210 \mu \mathrm{m})$

The proposed European contributions will consist of the telescope subsystem, the ground segment and one of the onboard instruments: the far infrared imaging spectrometer SAFARI (SpicA FAR infrared Instrument). The SAFARI instrument will be financed through national contributions. The Rutherford Appleton Laboratory (RAL) has been leading the Phase A study for this European SAFARI spectrometer. Currently SRON is taking over this leading role from RAL.

The SAFARI instrument is an imaging Fourier Transform Spectrometer (FTS) and contains an Optical Delay Line (ODL) scan mechanism: FTSM. TNO has been participating in a SPICA SAFARI study, led by SRON, to support the

*teun.vandendool@tno.nl; phone +31 15269 2957; fax +31 15269 2111; tno.nl

Modern Technologies in Space- and Ground-based Telescopes and Instrumentation, edited by Eli Atad-Ettedgui, Dietrich Lemke, Proc. of SPIE Vol. 7739, 77391B · @ 2010 SPIE · CCC code: 0277-786X/10/\$18 · doi: 10.1117/12.857676 
RAL SPICA SAFARI Phase A study. The objective of this study is to increase the overall SAFARI TRL and improve the chances for SPICA in the ESA Cosmic Vision down selection. TNO participates in the optical design trade off between the SAFARI instrument and the FTS scan mechanism and leads the preliminary design of the FTS scan mechanism. Micromega-Dynamics contributes to the magnetic bearing design. SRON is developing control electronics for the FTSM.

The basis for conducting this study is the successful design by TNO, Micromega-Dynamics, and SRON of a bread-board ODL for the DARWIN mission ${ }^{1}$. The DARWIN ODL has a 2-mirror cat's-eye optical layout with magnetic bearing guiding system and voice OPD actuator (see Figure 2).

This paper describes the result of a phase-A conceptual design study on the FTSM.

This work is supported by the Netherlands Space Office (NSO).

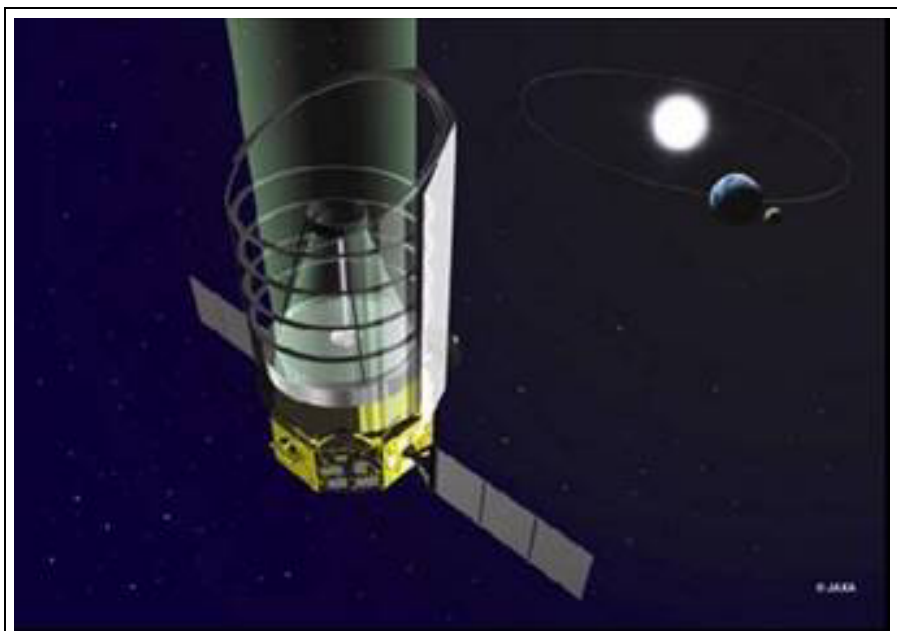

Figure 1 - The SPICA IR space observatory (courtesy JAXA).

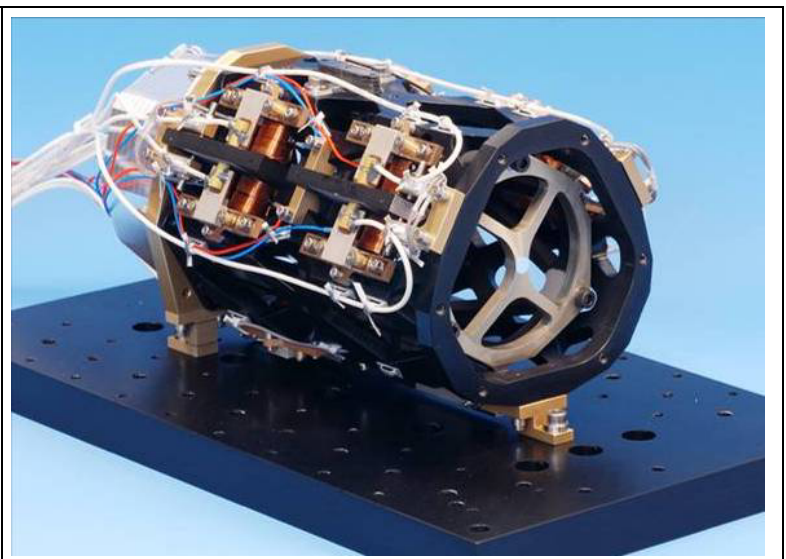

Figure 2 - The DARWIN Optical Delay Line with magnetic bearings. Heritage of this mechanism was used for the design of the SAFARI FTS mechanism.
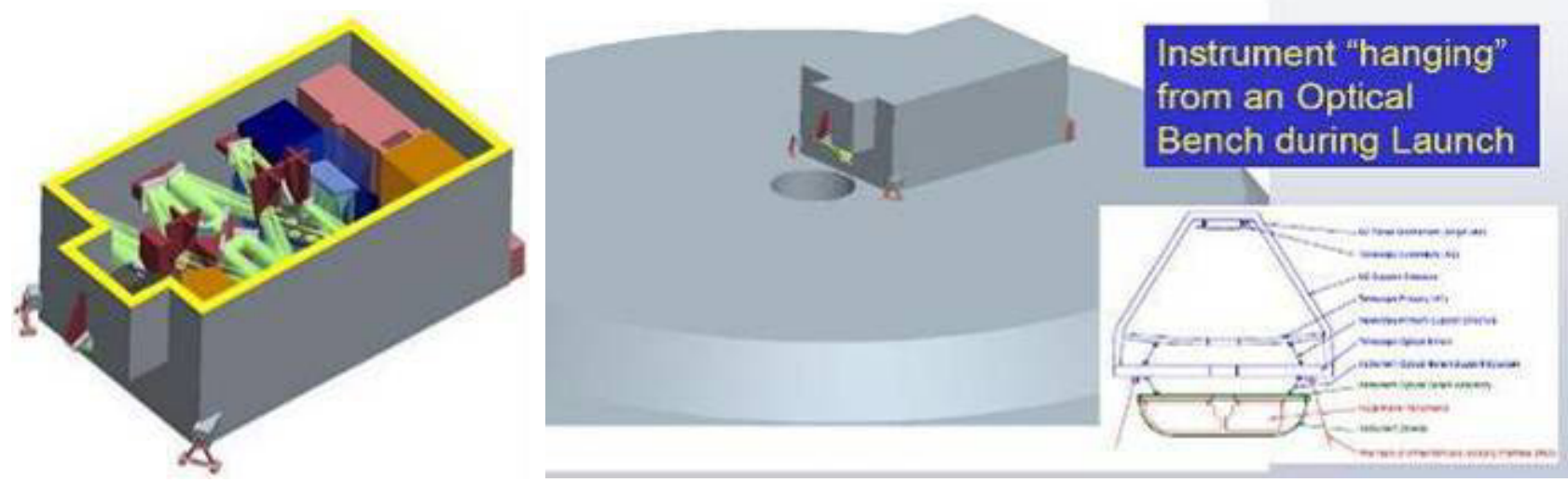

Figure 3 - The SAFARI instrument is one of the instruments 'hanging' below the optical bench in the focal plane of the primary mirror. 


\section{REQUIREMENTS}

SAFARI is one of the instruments on the SPICA optical bench. The optical bench is in the focal plane of the primary mirror, see Figure 3.

The requirements for the SPICA SAFARI FTS are influenced on, and an extension of, the Herschel/SPIRE spectrometer requirements ${ }^{2}$. The spectral resolution is in the same order $(30<\mathrm{R}<30000)$ but at smaller wavelengths: $30-207 \mu \mathrm{m}$ for SAFARI instead of 194-671 $\mu \mathrm{m}$ for SPIRE, giving rise to higher requirements on mechanical accuracies.

The most important requirements for the FTS mechanism are:

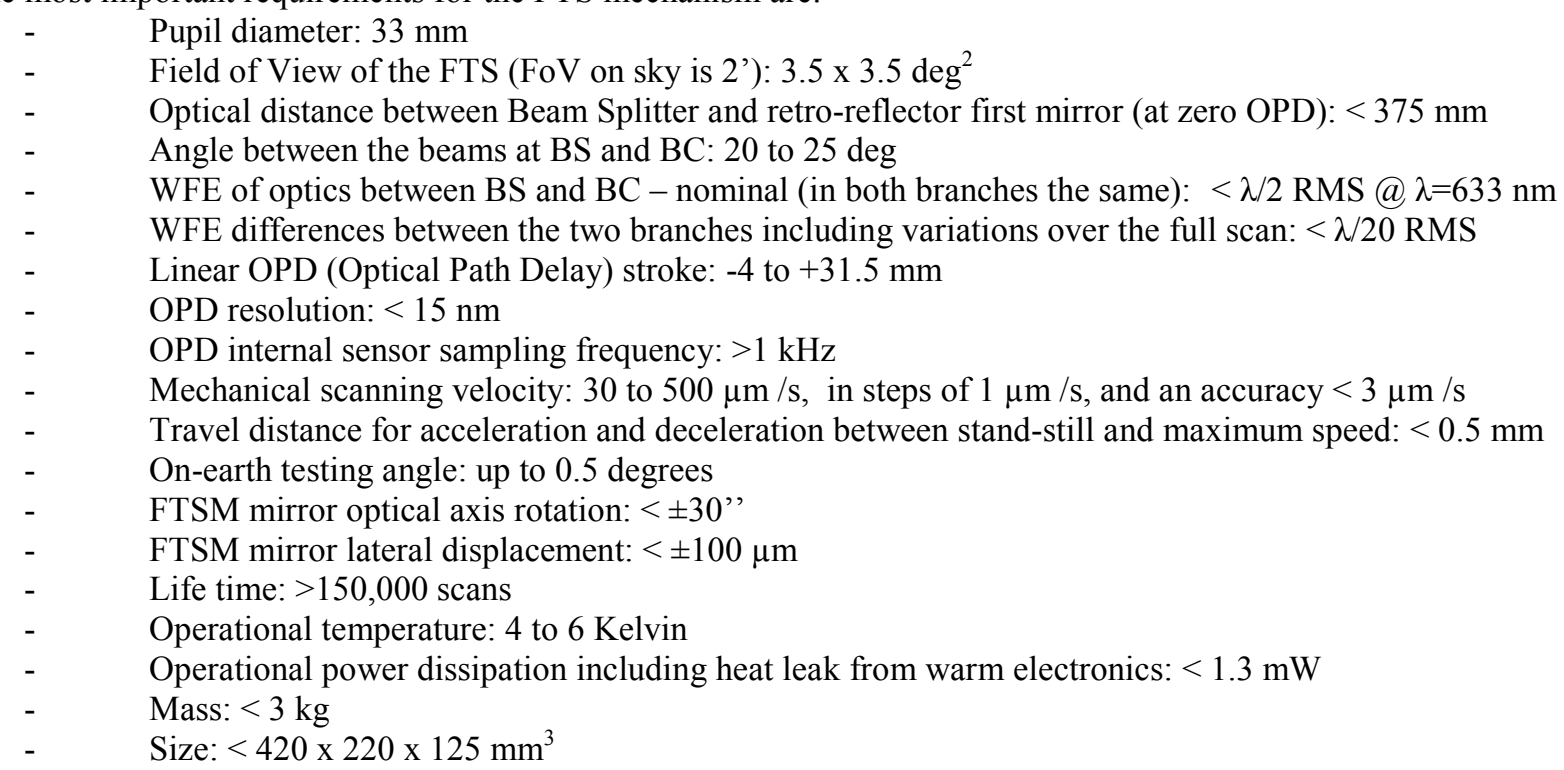

The conceptual design described in the next section is compliant with all these requirements.

\section{DESIGN}

\subsection{Optical configuration}

Figure 4 shows the optical configuration of SAFARI at the start of this project. It was based on HERSCHEL/SPIRE heritage. The delay line was drawn as two roof-top retro-reflectors in a back-to-back configuration. This was replaced by a design with two f-number $=4.5$ cat's-eyes because of the inherent pupil reimaging of a cat's-eye which allows for smaller Beam-Splitter (BS) and Beam-Combiner (BC), and therewith a smaller overall layout, matching the required dimensions and distance between BS and first reflecting surface of the retro-reflector. Another advantage is the less critical alignment, only the distance between M1 and M2 needs accurate alignment (to a few $\mu \mathrm{m}$ ). There is no mass penalty relative to other retro-reflector types. A disadvantage is the inherent nominal WFE (Wave Front Error) for the large required FoV (Field of View), but this was still within the required value of $<\lambda / 2$ at $2.5 \times 3.5 \mathrm{deg}^{2}$.

The combination of requirements on dimensions and mass, OPD stroke, pupil diameter, and distance between BS and retro-reflector first mirror made a back-to-back configuration of the two retro-reflectors impossible. Therefore a configuration was chosen with two non-coaxial (side-by-side) on-axis cat's-eyes. This also allowed for a central "backbone" tube in which all mechatronic guiding and driving components could be housed, leading to an overall tidy and compact design. The draw-back of this side-by-side design is that two extra folding mirrors are required (see Figure 5).

The optical layout between the FTS mechanism and the detectors (three cameras for three wavebands) was changed such that the detectors could be placed close together on a row, see Figure 6. This made the design compact and more easy to cool. 


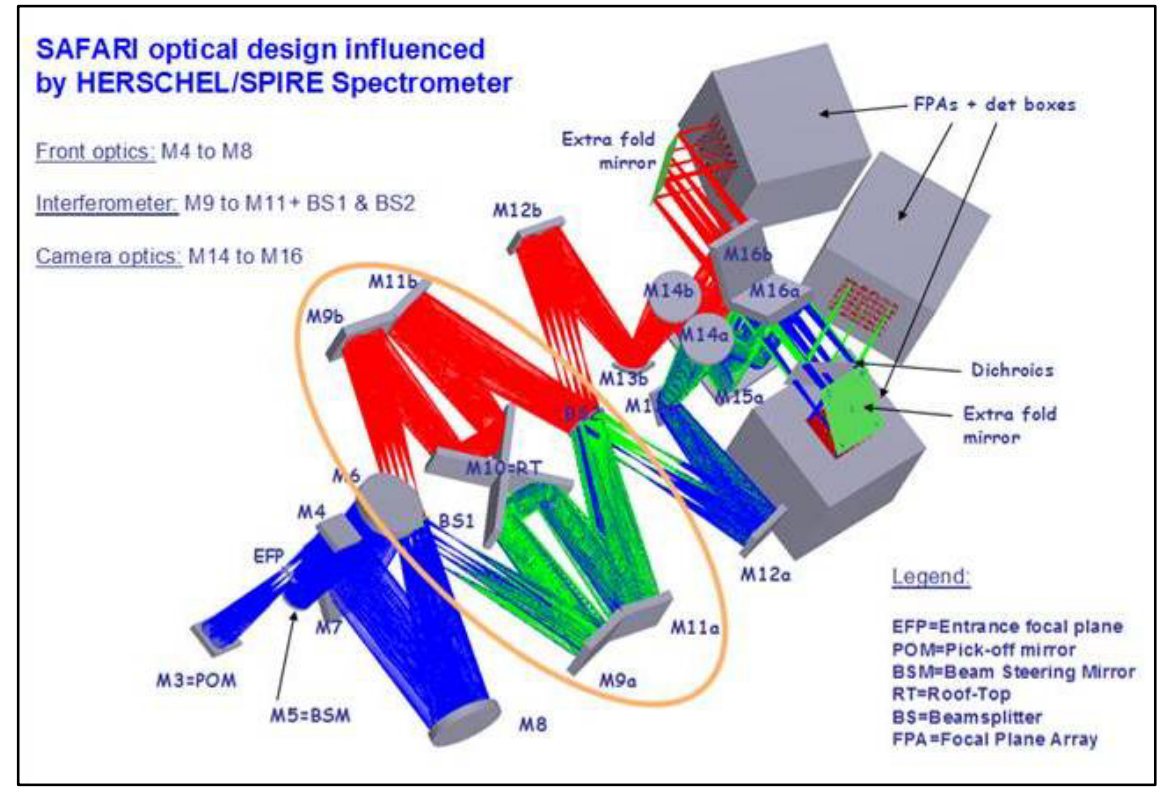

Figure 4 - Preliminary SAFARI optical layout with the FTS mechanism encircled. Here still with a roof-top retroreflector which was replaced by a cat's-eye design in this project.
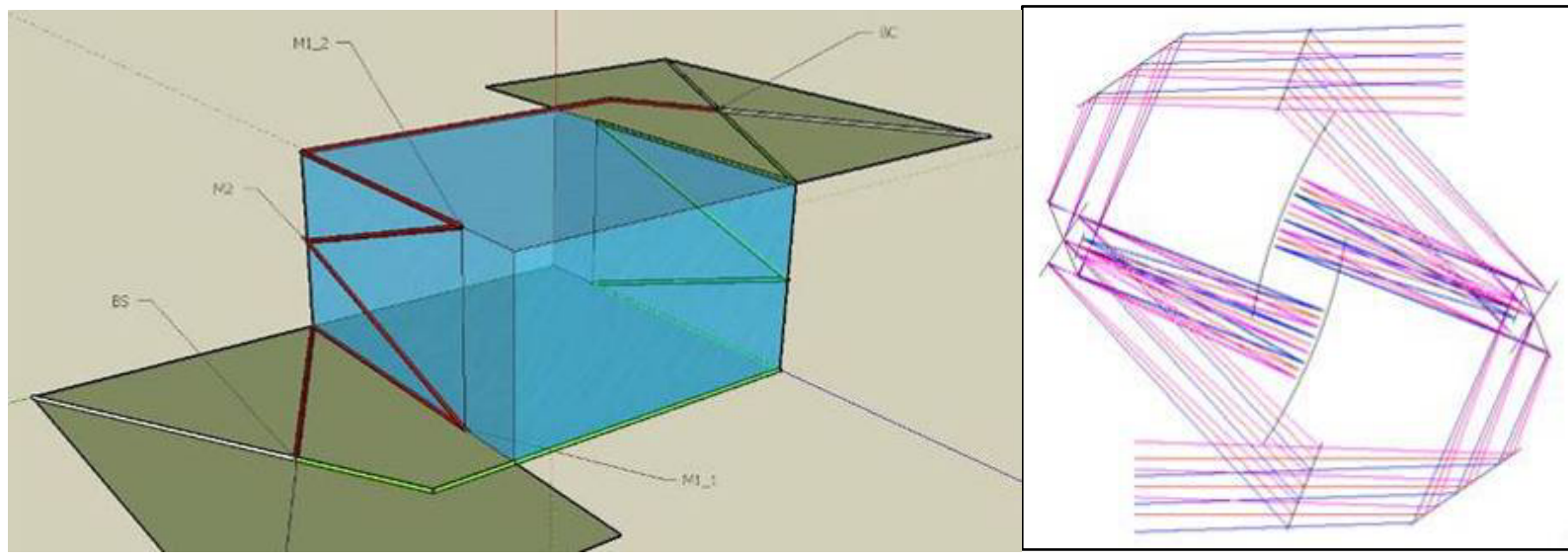

Figure 5 - Because of volume requirements, a stacked optical layout of the FTS was chosen using two cat's-eye retro-reflectors.

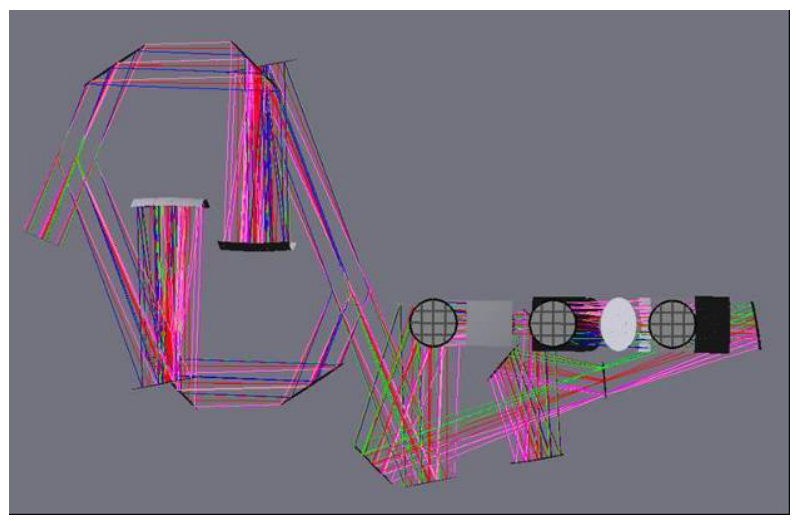

Figure 6 - SAFARI Optical layout including detectors. 


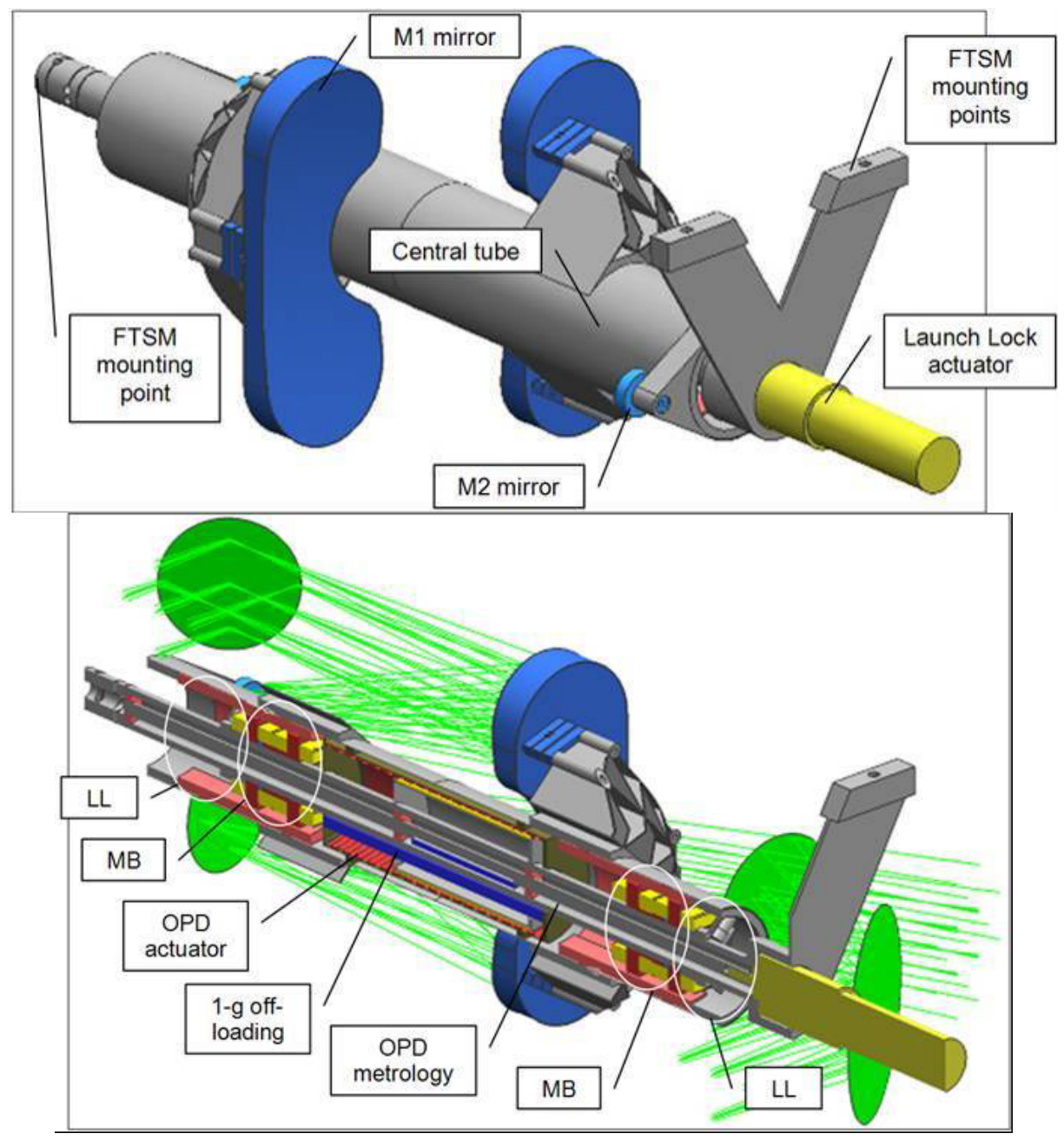

Figure 7 - The FTS mechanism consists of a central back-bone with all guiding and driving inside.

\subsection{Guiding mechanism}

There is not a large choice of guiding mechanisms that will meet the required large stroke, small volume, and low power. Similar requirements for the DARWIN ODL (Optical Delay Line) led to choosing an active magnetic bearing (MB), which was based on the MABE mechanism developed by Micromega Dynamics. The same magnetic bearing technology was used for the SAFARI FTS mechanism. An additional advantage is that the OPD resolution is only limited by metrology performance, because a magnetic bearing has no friction or other OPD performance limiting non-linearities.

In the DARWIN ODL the magnetic bearings were placed outside of the optics (see Figure 2). In the SAFARI FTS mechanism the bearings were put in between the optics inside a central "back-bone" tube (see Figure 7). This was a natural way of construction because of the side-by-side configuration of the retro-reflectors in the SAFARI FTS. All other opto-mechatronic parts could be placed inside the tube as well, such as: MB sensors, OPD actuator, a stepper motor operated Launch-Lock (LL), and OPD metrology. The back-bone design also leads to a high first eigen-frequency: $\sim 1000 \mathrm{~Hz}$. The central tubular structure including mirror mounts is designed such that it can be manufactured by spark erosion out of a single piece of Aluminum, which makes the system a-thermal so the optics are automatically in focus at any temperature.

Figure 8 shows the active magnetic bearing layout. Two of these sets, one at either side of the central tube, constrain 4 degrees of freedom (DoF). The fifth degree of freedom (rotation around the central axis; Rz) is only constrained in a 
weak and passive way. It is still under investigation if this is sufficient or if one of the MB sets has to be replaced by a 3-DoF type. This MB design can be made fully redundant by only modifying the wiring and accompanying electronics.

The power dissipation inside the FTS mechanism is negligible in space (zero-gravity environment). The heat leak through the wires can form a considerable part of the total FTS mechanism power budget especially if the magnetic bearings also need to carry the 1-g forces. Therefore during earth testing (1-g environment) some measure is needed to keep the power dissipation low. In Figure 7 one option for 1-g offloading is indicated with a permanent magnet that is turned by the launch-lock motor such that on earth it is supplying an extra lifting force, and in space it is turned such that it is shunted and doesn't supply a force. Other options include:

Increase the size of the vertical MB relative to the horizontal ones, making them stronger at the same power dissipation.

- $\quad$ Modify the MB magnetic circuit to increase vertical negative stiffness such that a small vertical rotor displacement leads to the required extra 1 -g force.

Which of these options will be the most optimal is still under investigation.

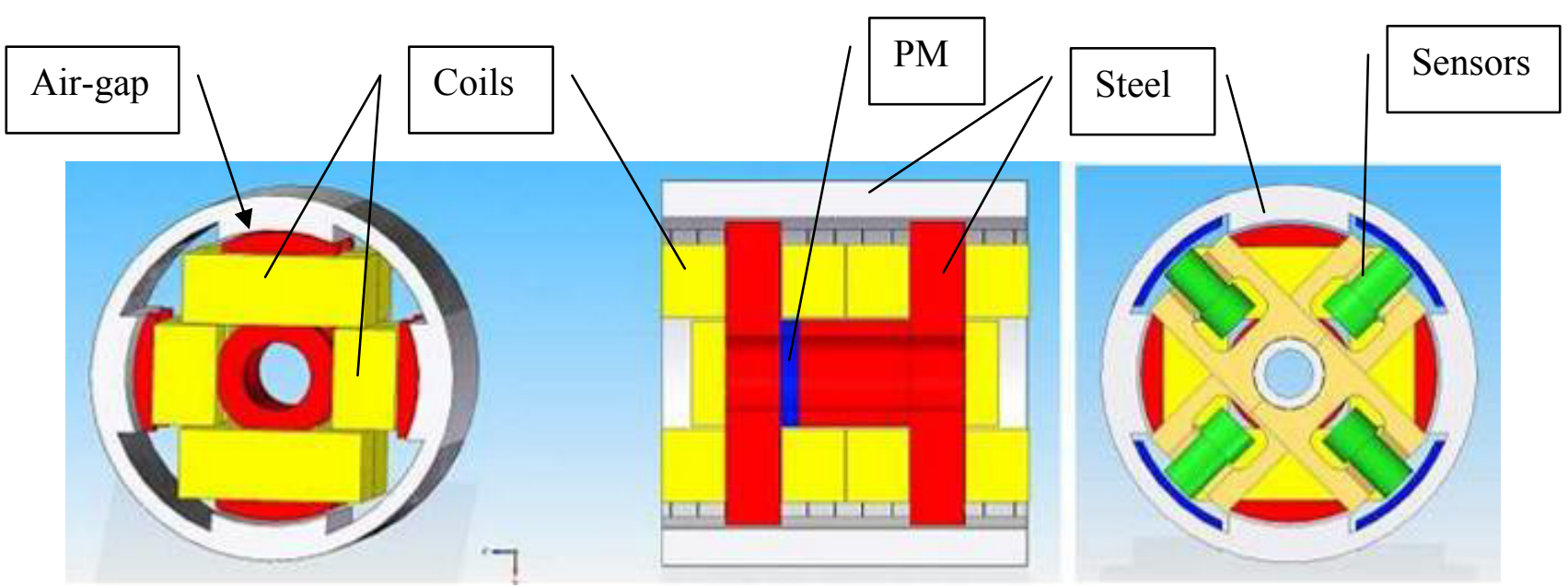

Figure 8 - One of the two active differential radial magnetic bearings. Red and white are steel parts, the coils are yellow and the permanent magnet (PM) is blue. In the right picture the four differentially used Eddy-current sensors (green) are included "looking" at aluminum targets (blue). The outside diameter is $42 \mathrm{~mm}$.

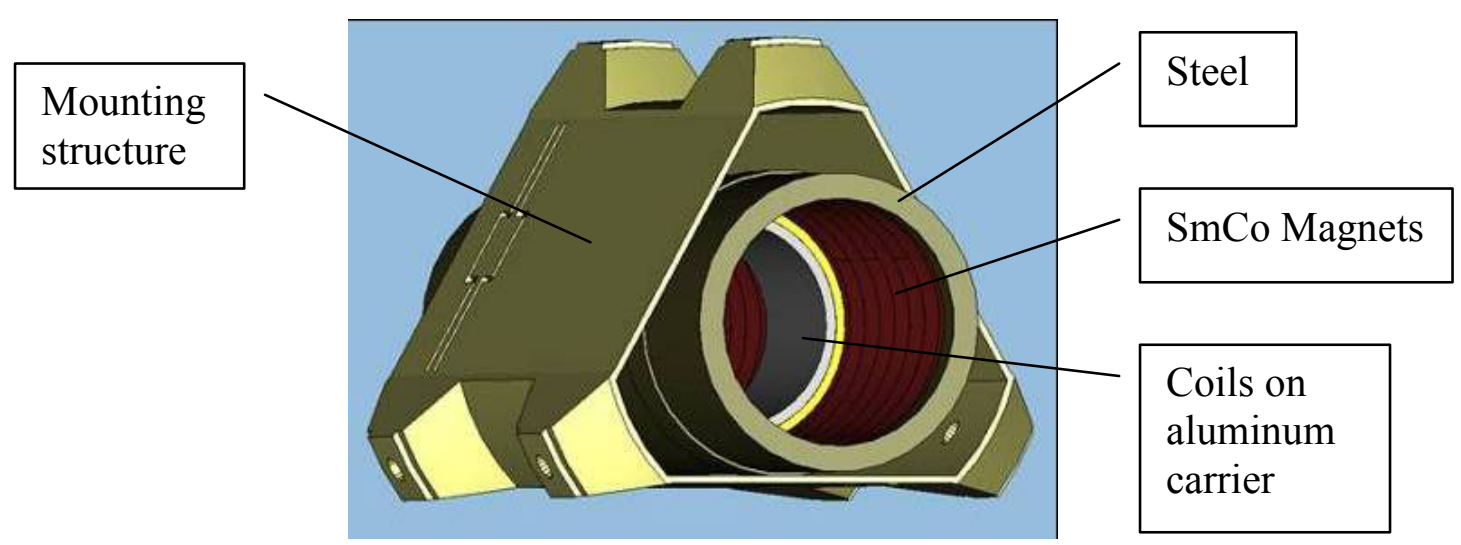

Figure 9 - SAFARI linear motor OPD actuator. 


\subsection{OPD actuator}

For the required large stroke and low power dissipation a voice-coil type of actuator like in the DARWIN ODL wasn't sufficient. A moving-coil linear motor design was chosen despite the needed more complex design, 3-phase control and additional wiring. Figure 9 shows the OPD actuator design. The total length of the motor (magnets) is $56 \mathrm{~mm}$ at a diameter of $22 \mathrm{~mm}$.

The driving requirement for the OPD actuator force is the ability to operate on earth at an angle of 0.5 degrees. Because of the $\sim 1 \mathrm{~kg}$ moving mass this leads to a horizontal gravitational force of $0.1 \mathrm{~N}$. This far exceeds the acceleration and deceleration requirements. With a linear motor this force can be generated at a moving (coil) mass of 100 gram and a stationary (magnet) mass of 300 gram. The power dissipation at cryogenic temperatures is below $0.1 \mathrm{~mW}$.

This OPD linear motor design doesn't set a limit on speed and resolution of the rotor position because of the inherent frictionless and stepless behavior. The performance is only limited by the OPD metrology.

\subsection{OPD Metrology}

At a stroke of more than $35 \mathrm{~mm}$ and a resolution of $15 \mathrm{~nm}$ (dynamic range $=2.4 \mathrm{e} 6$ ) only counting type of sensors will comply. Such include magnetic, capacitive, inductive or optical linear scales (as in SPIRE). A fiber optic interferometer ${ }^{3}$ was selected as the baseline metrology system (see Figure 10). It is very small such that it can be mounted inside the central tube. Also the power dissipation inside the cold mechanism is negligible. Fiber optic interferometers currently are starting to be used in commercial applications, also in cryogenic-vacuum environments. Space compatibility including connectors, radiation hardness, and vibration and shock resistance have been reported independently as being viable.

The resolution is in the order of $1 \mathrm{~nm}$ or lower and the absolute accuracy can be in the order of $10 \mathrm{~nm}$. The band-width can be easily made in the order or $10 \mathrm{kHz}$. Stray-light has to be shielded because the SAFARI detectors are very sensitive.

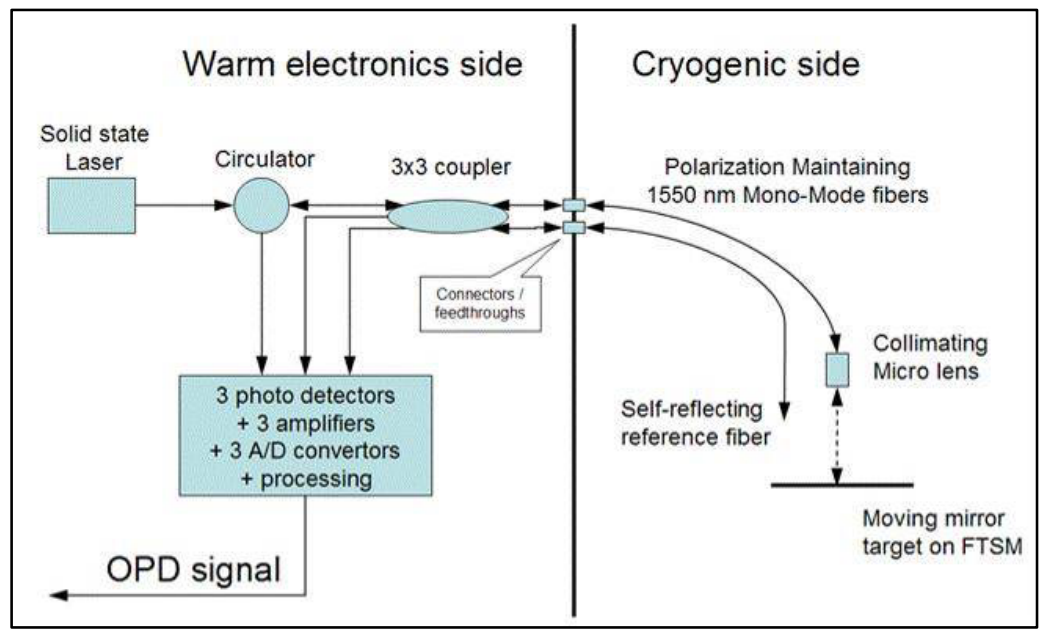

Figure 10 - Schematic of the SAFARI fiber interferometer internal OPD metrology.

\subsection{Electronics}

In the framework of the Darwin ODL project, SRON developed an FPGA based combined MB/OPD controller "ODELCO" with a sampling/control rate of $10 \mathrm{kHz}$ and a targeted power dissipation of $5 \mathrm{~W}$ (see Figure 11 and Figure 12). It is further being developed for other applications including SAFARI. It is proposed as a baseline for control of the FTSM. 


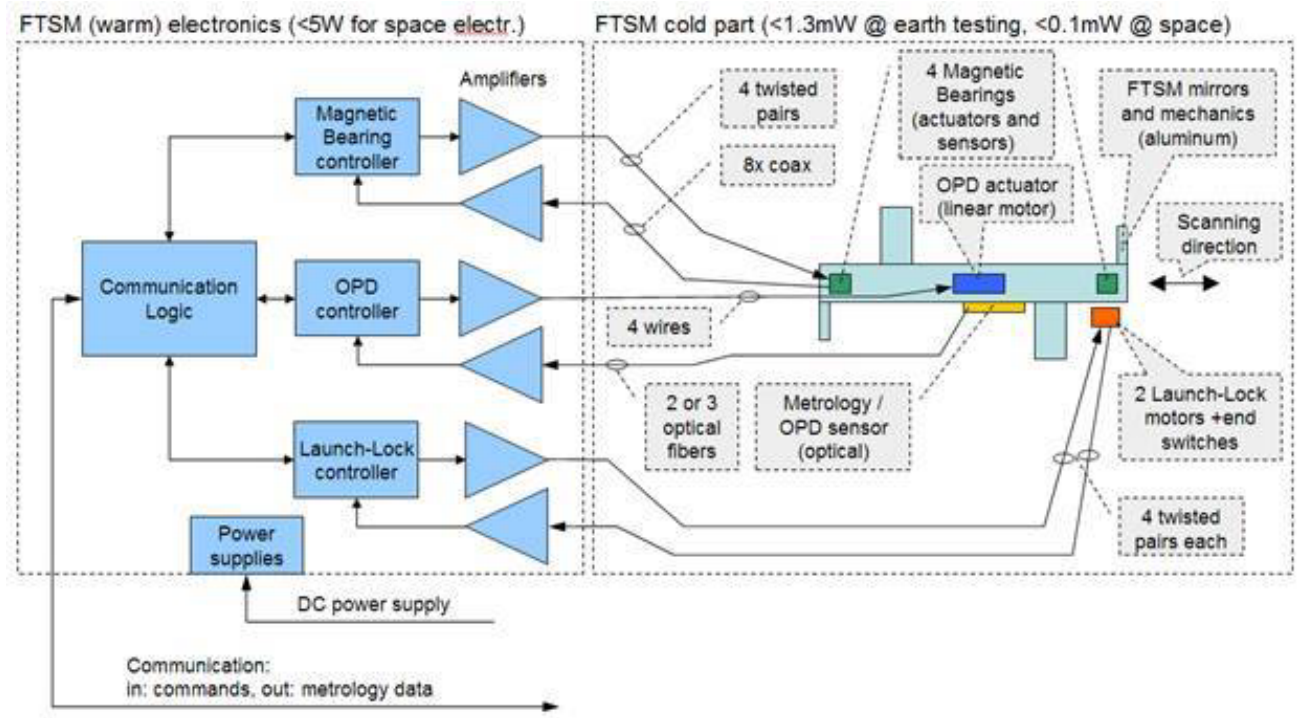

Figure 11 - Schematic of the SAFARI electronics.

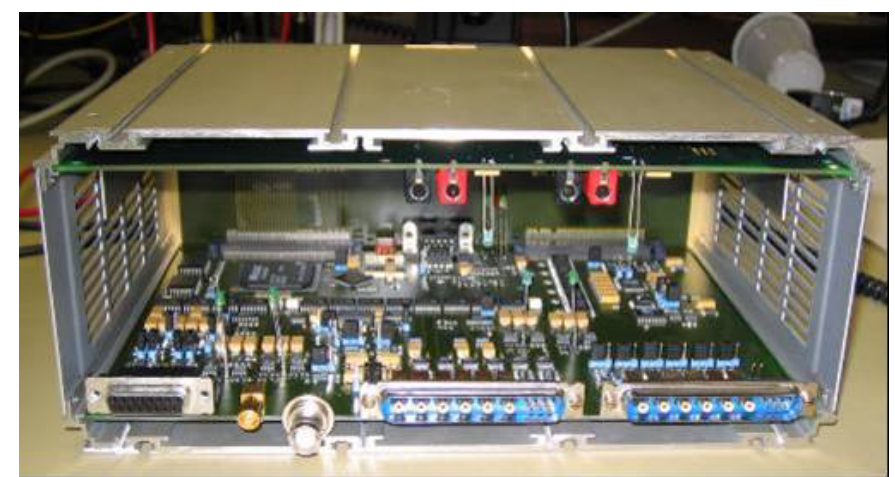

Figure 12 - Inside view of the ODELCO FPGA based MB and OPD controller. Lower board: analogue electronics including ADC, DAC, encoder interface and power; upper board: digital electronics including FPGA, RS-485, and CAN.

\section{CONCLUSIONS}

An ODL design has been realized that fulfils all SPICA SAFARI FTS mechanism requirements. Future development that will be carried out include:

- Adaptation to optical updates

- Optimization of magnetic bearing (for $1 \mathrm{~g}$ operation)

- Minimization of power dissipation and heat leak

- Design of the launch lock

- Further development of the fiber interferometer

- Implementation of a breadboard version of the FTS mechanism to be used for SAFARI evaluation

\section{REFERENCES}

[1] T.C. van den Dool, et al. "The development of a breadboard cryogenic optical delay line for Darwin", Proc. SPIE 6692, pp.66920A.1-66920A.12 (2007).

[2] Matt Griffin, et al., "Herschel-SPIRE: Design, Ground Test Results, and Predicted Performance", Proc. SPIE 7010, pp.701001-701006 (2008).

[3] L.K. Cheng, et al., "Development of a fringe sensor based on 3x3 fiber optic coupler for space interferometry", Proc. SPIE 5855, pp.1004-1007 (2005). 